\title{
DOI: https://doi.org/10.24297/jap.v16i1.8192
}

\section{Spectroscopy and Conductivity Studies of Polyvinyl Alcohol (PVA)/Polypyrrol (Ppy) Nanocomposite with Various Chloride Metals to Improved Properties of the Polymers}

\author{
N.A.M. Shahin ${ }^{1}$, R.K. Abd El Hamid ${ }^{1}$. \\ Physics Department, Faculty of Women for Arts, Science and Education, Ain Shams University, Cairo, \\ Egypt,11577.
}

\begin{abstract}
Polyvinyl alcohol- polypyrrole (PVA-PPY) nanocomposites with metal chlorides ( $\mathrm{FeCl}_{3}, \mathrm{NiCl}_{2}, \mathrm{CuCl}_{2}$ and $\mathrm{ZnCl}_{2}$ ) have been synthesized by chemical oxidative polymerization method. These synthesized nanocomposites are characterized by using FTIR, X-Ray Diffraction, Transition Electron Microscope (TEM) and Conductivity measurement. TEM exhibit that all of the composites have uniform sizes and morphologies. The diameter of PVA/PPY nano composite is 58nm when the metals added to the PVA/PPY the diameters becomes smaller. The variation of electrical conductivity ( $\log \sigma$ ) with 1000/T for PVA/PPY nanocomposite with metal chlorides revealed that the increase in conductivity $\sigma$ at temperature (393K) with added metals can be attributed to the creation of induced charge carriers in PVA/PPY matrix
\end{abstract}

Key Words: Polyvinyl alcohol, polypyrrole, metal chlorides, FTIR, X-Ray diffraction, Electrical properties

\section{INTRODUCTION}

Crystallization behavior of semi crystalline polymer has been extensively investigated due to their technological and theoretical importance. Diverse semi crystalline polymers such as polyethylene (PE), polypropylene (PP), polyamide, poly (ethylene terephthalate) (PET), and polystyrene (PS), have been adopted to analyze the crystallization behavior of polymeric materials. L Pan et al [1]

A vinyl polymer, namely polyvinyl alcohol (PVA) is semi crystalline, water soluble, and low electrical conductivity material. PVA exhibits certain physical properties resulting from crystal-amorphous interfacial effects. Electrical conductivity of PVA can be tailored to a specific requirement by the addition of suitable material. Crystallization behavior of poly (vinyl alcohol) (PVA) has been also studied by several research groups under different experimental conditions because the hydroxyl group containing semi-crystal polymers have been of special interest. $\mathrm{H}$ Byun et al [2]. In general, these studies have been conducted using DC electrical conductivity, X-ray different scattering (XRD), Fourier transform infrared spectroscopy (FTIR) and Transition electron microscopy (TEM).

Among all the conducting polymers, polypyrrole (PPY) has received much attention since the monomer pyrrole is easily oxidized, water soluble and commercially available. Although pyrrole is capable of produce conducting polymers with high electrical conductivity, environmental stability and good redox properties. $\mathrm{H} \mathrm{N}$ M Ekramul Mahmud et al [3].

Polypyrrole (PPY) is a conducting conjugated polymer, has attracted much interest due to its low cost, easy synthesis, good stability, and environmentally benign performance. D M Nerkar [4].The conductivity of a conductive polymer is strongly dependent on the doping agents with electron donor or acceptor abilities. The doping process can even transform an intrinsically conjugated polymer insulator to a near - metallic conductor. $\mathrm{K}$ Lee et al [5]. 
In the present work, the Polyvinyl/ polypyrrole (PVA/PPY) Nan composite with metal chlorides $\left(\mathrm{FeCl}_{3}, \mathrm{NiCl}_{2}\right.$, $\mathrm{CuCl}_{2}$ and $\mathrm{ZnCl}_{2}$ ) have been characterized by using various techniques such as FTIR, XRD, TEM and DC electrical conductivity.

The XRD and TEM are performed in order to confirm the crystallization behavior of PVA/PPY metals Nan composite. In this regard we have made an attempt to study the electrical conductivity of pure PVA, PPY and nanocomposite with metals to know the influence of metals on pure polymer in answering better electrical conductivity.

\section{EXPERIMENTAL}

\subsection{Materials:}

Polyvinyl alcohol (PVA) (Mw:6000), INDIA, and pyrrole (PY), Germany, monomer supplied by were obtained and used in the present study pyrrole monomer was purified by distillation under reduced pressure and stored at $4^{\circ} \mathrm{C}$ in the absence of light. Analytical grade ammonium per sulphate (APS) (MW: 228.19), INDIA was used as oxidizing agent.

\section{2: Synthesis of PVA/PPy and PVA/PPy metals chloride nanocomposite:}

A Solution containing $3 \mathrm{gm}$ of PVA in $100 \mathrm{ml}$ distilled after was prepared by constant stirring and heating the mixture up to $90^{\circ} \mathrm{C}$ for an hour. Then the mixture was left to cool down to room temperature while the stirring of the mixture was carried out to ensure a homogenous composition. $2.013 \mathrm{gm}$ of pyrrole monomer was added to the solution of $3.42 \mathrm{gm}$ of ammonium per sulfate $\left[\left(\mathrm{NH}_{4}\right)_{2} \mathrm{~S}_{2} \mathrm{O}_{8}\right]$ and this reaction mixture was stirred continuously for 3 hours at a constant temperature $\left(5^{\circ} \mathrm{C}\right)$ to obtain polypyrrole. $100 \mathrm{ml}$ of PVA solution was mixed with $100 \mathrm{ml}$ of PPY solution in 1:1 ratio by volume stirred for 20 minutes at the room temperature in order to get homogenous solution. Finally $0.3 \mathrm{gm}$ of $\mathrm{FeCl}_{3}$ was added in the mixture and stirred for 12 hours. By repeat the last step with added $0.3 \mathrm{gm}$ of $\mathrm{NiCl}_{2}, \mathrm{CuCl}_{2}$ and $\mathrm{ZnCl}_{2}$ respectively. The obtained product was filtered or center fused and washed thoroughly with methyl followed by distilled water. Nanocomposite was vacuum dried at $60^{\circ}-70^{\circ} \mathrm{C}$ for $1 \mathrm{~h}$.

\section{MEASUREMENTS}

A Fourier transforms infrared (FT- IR) spectrometer (Model Jasco, 300E). The IR spectrometer in $\mathrm{KBr}$ medium at room temperature in the region $4000-400 \mathrm{~cm}^{-1}$. The $X$-ray diffraction pattern of the sample was measured using (X PERT - PRO - PAnalytical). XRD operating at $30 \mathrm{~mA}$ and $45 \mathrm{kv}$, using Cuk(alpha), $\left(\lambda=1.4506 \mathrm{~A}^{\circ}\right)$. The diffract gram was recorded in terms of $2 \theta$ in the range $(0-100)^{\circ}$. The powder of morphology the nanocomposite was investigated by TEM (JEOL JEM. 1010). The nanocomposite powder was prepared by making a suspension from the powder in distilled water. The suspension was centrifuged to collimate the large size particles. To study the electrical conductivity of nanocomposites powder it should be pressed in form of circular pellets with $13 \mathrm{~mm}$ diameter and $1-3 \mathrm{~mm}$ in thickness by applying pressure $10 \mathrm{tons} / \mathrm{cm}^{2}$. The pellets of PVA/PPY and its metal nanocomposites were coated with silver paste on either side. The DC electrical conductivity of pure PVA/PPY and metal nanocomposite was investigated over the temperature range from room temperature to about 393K to study the effect of metals on the D.C electrical properties of PVA/PPY. The electrical conductivity $\sigma$ was calculated using:

$\sigma=\frac{L}{R A}$, where $\mathrm{R}=$ electrical resistance of the disc (ohm), $\mathrm{A}=$ surface area of the disc $\left(\mathrm{cm}^{2}\right), \mathrm{L}=$ thickness of the disc $(\mathrm{cm})$ 


\section{RESULTS and DISCUSSION}

\subsection{FTIR Spectra}

The FTIR spectra of synthesized PVA/PPY and PVA/PPY nanocomposite with $\left(\mathrm{FeCl}_{3}, \mathrm{NiCl}_{2}, \mathrm{CuCl}_{2}\right.$ and $\left.\mathrm{ZnCl}_{2}\right)$ are shown in Fig (1: a, b, c, d, e). Figure (1: a) shows FTIR spectrum of PVA/PPY. The bands appear in the region $3300-3500 \mathrm{~cm}^{-1}$ due to $\mathrm{N}-\mathrm{H}$ stretching frequency of an aromatic amine, Q Adeloju et al [6]. A broad band observed at $3418 \mathrm{~cm}^{-1}$ in the spectrum of PVA/PPy powder and $\mathrm{NH}$ region also shows dependence of the doping anion of organic acids. Anion which typically forms hydrogen bond with amine group shows variations in the intensity and shape of the $\mathrm{NH}$ band, thus indicating that the doping is higher in the sample. The bands at $2919 \mathrm{~cm}^{-1}$ and $2955 \mathrm{~cm}^{-1}$ are due to $\mathrm{CH}_{3}$ and $\mathrm{CH}_{2}(\mathrm{C}-\mathrm{H}$ stretching).

The two bands observed in the range $1400-1467 \mathrm{Cm}^{-1}$ are due to the stretching of $\mathrm{C}-\mathrm{N}$ frequency of the benzonic and quinonic rings respectively because of the conducting state of polymer, DB Dupare et al and $\mathrm{H}$ Bai et al $[7,8]$. The bands corresponding to quinoid $(N=Q=N)$ and benzenoid $(N-B-N)$ ring stretching are observed at $1559 \mathrm{~cm}^{-1}$ for $(C=N)$ stretching and $1311 \mathrm{~cm}^{-1}$ for $(C-N)$ respectively. The band observed at 1047 $\mathrm{cm}^{-1}$ is due to the symmetric and asymmetric $(C-O)$ stretching vibrations of polyvinyl group, and the peaks observed at $675 \mathrm{~cm}^{-1}$ are due to $(\mathrm{C}-\mathrm{H})$ bending.

All these observations support the presence of conducting emeraldine salt phase of PVA/PPY composites materials. MF Mabrooket al and DJ Shirale et al $[9,10]$.

Figures (1: b, $c$, d, e) shows FTIR spectrum of PVA/PPY nanocomposite with $\left(\mathrm{FeCl}_{3}, \mathrm{NiCl}_{2}, \mathrm{CuCl}_{2}\right.$ and $\mathrm{ZnCl}$ ) respectively which exhibits absorption peaks at $(3341,3320,3310$ and 3390$) \mathrm{cm}^{-1}$ for $\mathrm{N}-\mathrm{H}$ stretching of aromatic amine. The two bands at $1559 \mathrm{~cm}^{-1}$ and $1311 \mathrm{~cm}^{-1}$ for quinoid and benzenoid ring stretching modes for $C=N$ and $C-N$ shifted to $\left(1587,1557,1588\right.$ and 1551) $\mathrm{cm}^{-1}$ and $\left(1328,1302,1383\right.$ and 1301) $\mathrm{cm}^{-1}$ respectively. The presence of metal chlorides in the nanocomposites is strong supported by new peaks at

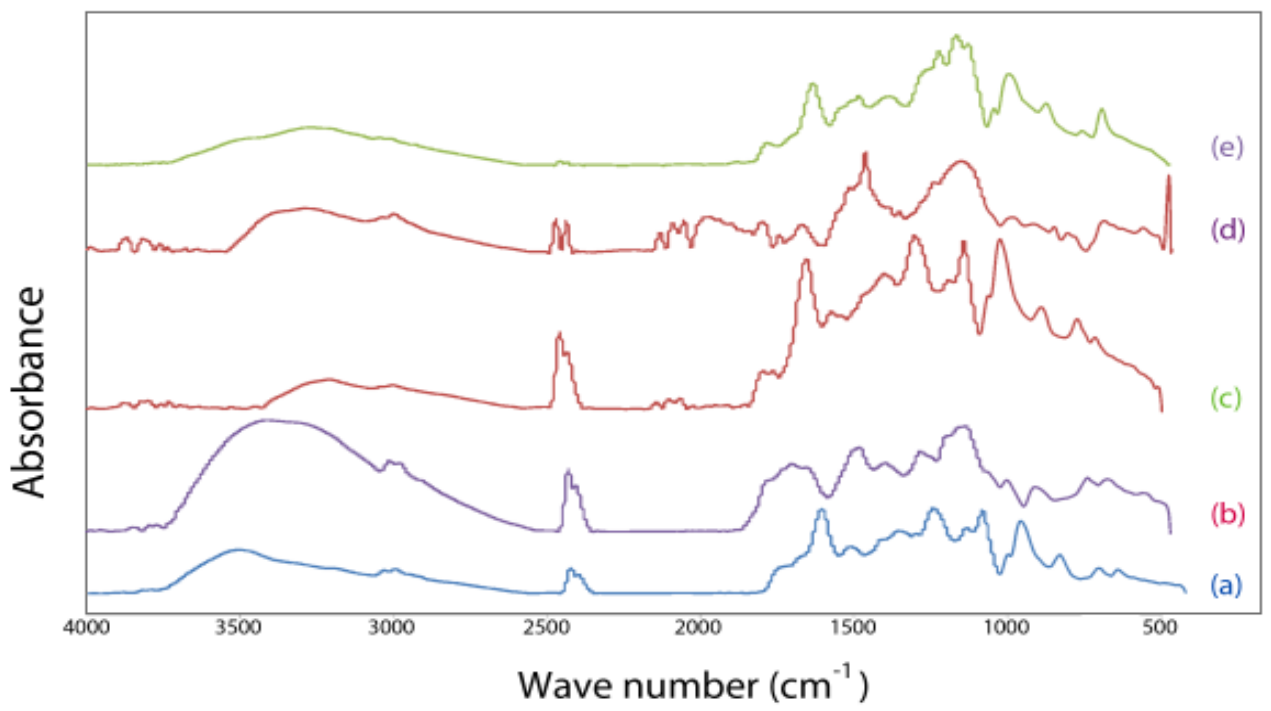

$(420,412,405$ and 428$) \mathrm{cm}^{-1}$. The appearance of these bands indicates that the metal chlorides interacted with PVA/PPY composite.

Figure 1: FTIR of (a) PVA/ PPY uncapped (b) PVA/ PPY composite with $\mathrm{FeCl}_{3}$ (c) PVA/ PPY composite with $\mathrm{NiCl}_{2}$ (d) PVA/ PPY composite with $\mathrm{CuCl}_{2}(\mathrm{e}) \mathrm{PVA}$ / PPY composite with $\mathrm{ZnCl}_{2}$. 


\subsection{X-Ray Diffraction}

Figure (2: a) presents X-ray diffraction pattern for uncapped PVA/PPY which exhibit strong diffraction peak for PVA at about $2 \theta=20^{\circ}$ correspond to the PVA crystalline phase, T Trindade et al [11], and exhibit a peak at about $2 \theta=24.6^{\circ}$ a characteristic peak of amorphous polypyrrole, Kyung Jin Lee et al [12] .
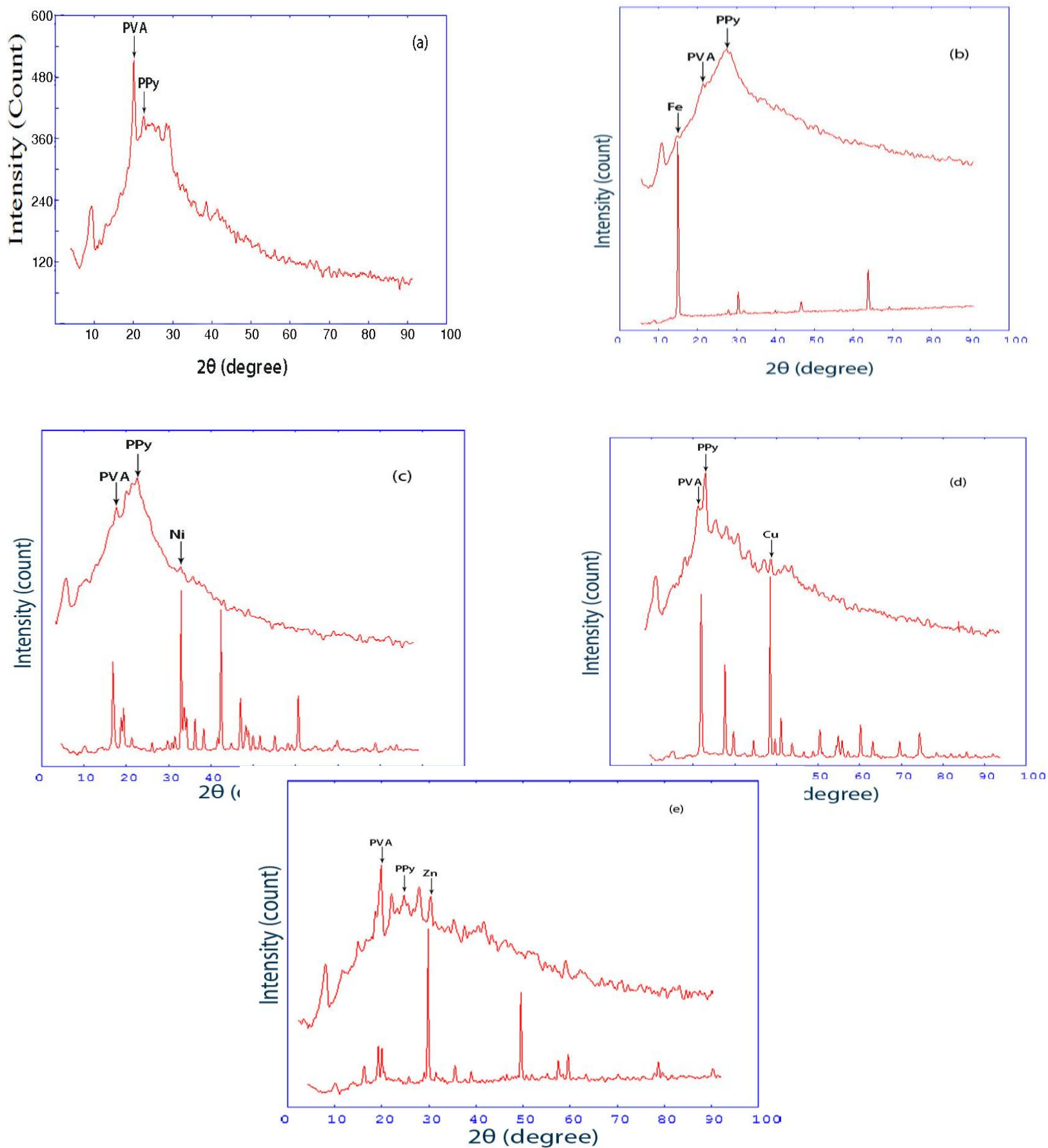

Figure 2: XRD of (a) PVA/ PPY uncapped (b) PVA/ PPY composite with $\mathrm{FeCl}_{3}$ (c) PVA/ PPY composite with $\mathrm{NiCl}_{2}$ (d) PVA/ PPY composite with $\mathrm{CuCl}_{2}(\mathrm{e}) \mathrm{PVA} / \mathrm{PPY}$ composite with $\mathrm{ZnCl}_{2}$. 
Figure (2: b,c,d,e) presents XRD pattern of pure metal chlorides and PVA/PPy capped with metals. The pattern indicates the crystalline nature of chloride metals. In this spectrum one main peaks appeared around $2 \theta=$ $15.5^{\circ}, 32^{\circ}, 33^{\circ}, 29^{\circ}$ for $\mathrm{FeCl}_{3}, \mathrm{NiCl}_{2}, \mathrm{CuCl}_{2}$ and $\mathrm{ZnCl}_{2}$ respectively. The $\mathrm{X}$-ray diffraction pattern of nanocomposites show that the intensity of diffraction peaks was decreased and broad. This attributed to the capped between PVA/PPy nanocomposites with metal decreased the crystallinity of metal chlorides. Also, it is confirmed that the metal chlorides retained those structures even though it's dispersed in PVA/PPy during polymerization reaction, Chivukula Srikanth et al [13].

\subsection{Transmission Electron Microscope (TEM)}

The size and shape of synthesized PVA/PPy nanocomposite with $\mathrm{FeCl}_{3}, \mathrm{NiCl}_{2}, \mathrm{CuCl}_{2}$ and $\mathrm{ZnCl}_{2}$ were determined by TEM. Figure (3: $a, b, c, d, e)$ presents the TEM images of nanocomposite materials. All of these composites present uniform sizes and morphologies. Figure (3: a) shows the PVA/PPy with an average diameter 58nm when the metals added to the PVA/PPy as in figure $(3: b, c, d, e)$ diameter of the nanocomposite is (22.96, $34.15,19.29$ and 19.24$) \mathrm{nm}$ respectively the particle size of nanocomposite becomes smaller this attributed to the effect of large particles size of polymer on the particle size of metals, due the decrease in ionic radius of metals which produce an agglomeration in TEM image.
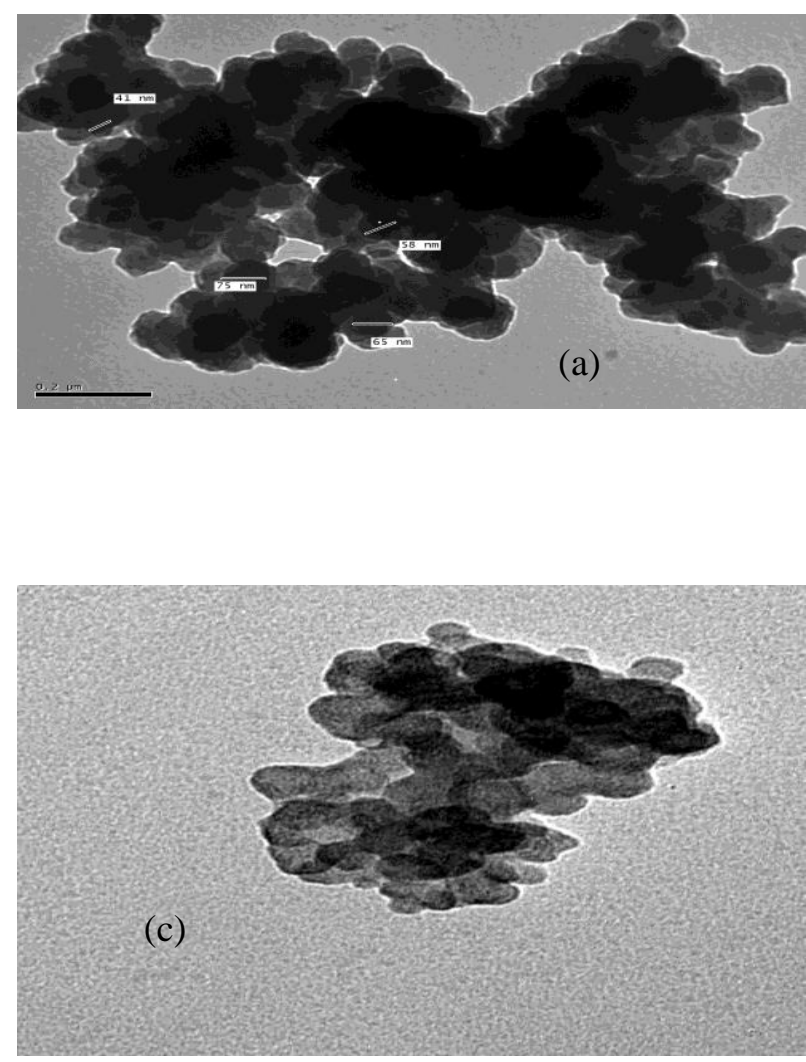
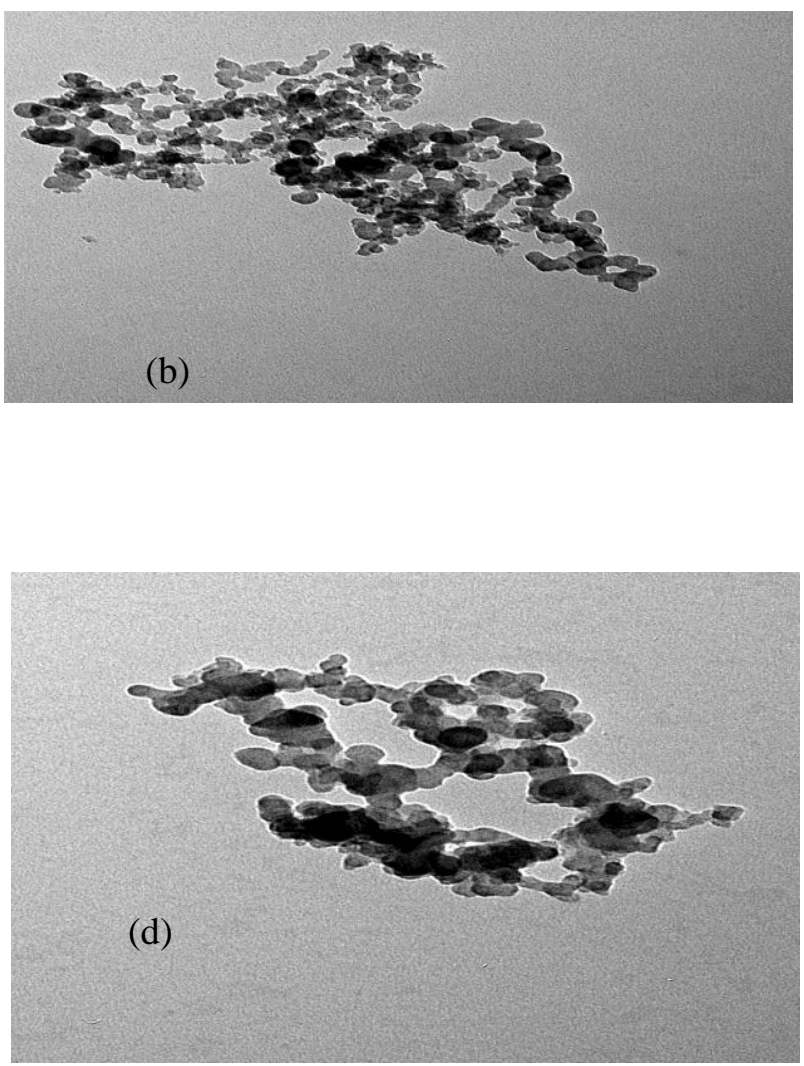


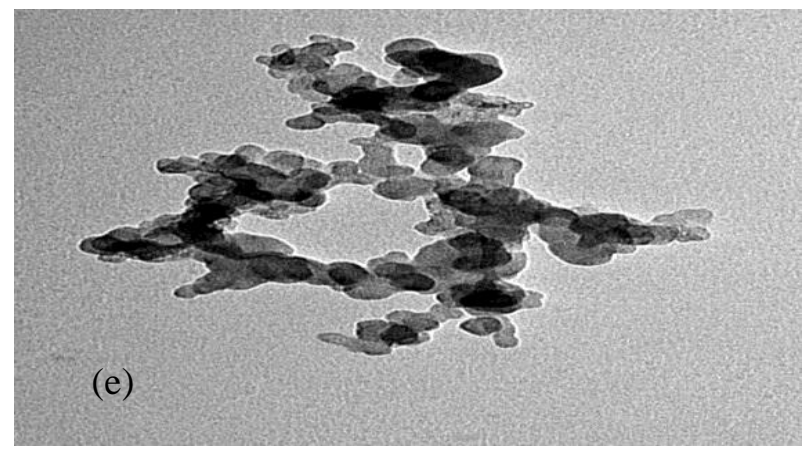

Figure 3:Transmission electron microscope (TEM) of (a) PVA/ PPY uncapped (b) PVA/ PPY composite with $\mathrm{FeCl}_{3}$ (c) PVA/ PPY composite with $\mathrm{NiCl}_{2}$ (d) PVA/ PPY composite with $\mathrm{CuCl}_{2}(\mathrm{e})$ PVA/ PPY composite with $\mathrm{Zncl}_{2}$.

\subsection{Electrical Conductivity}
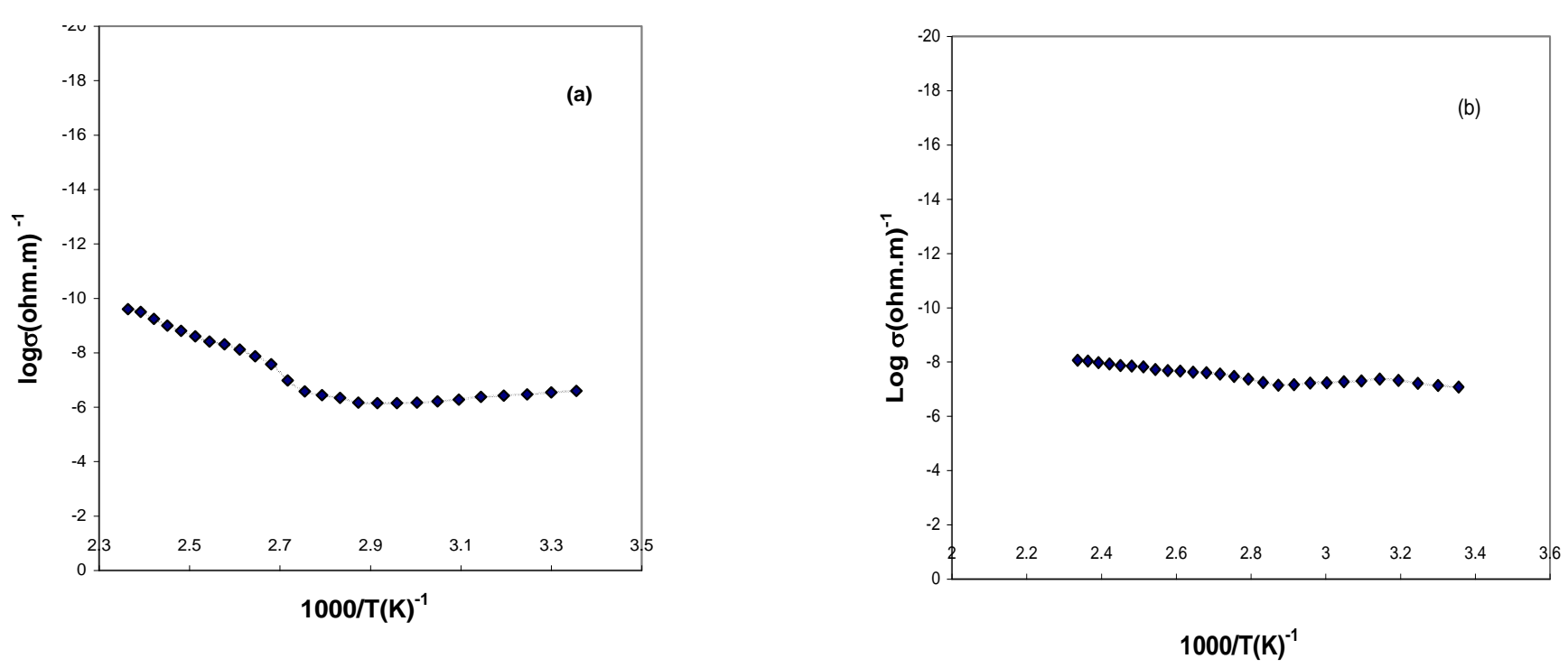

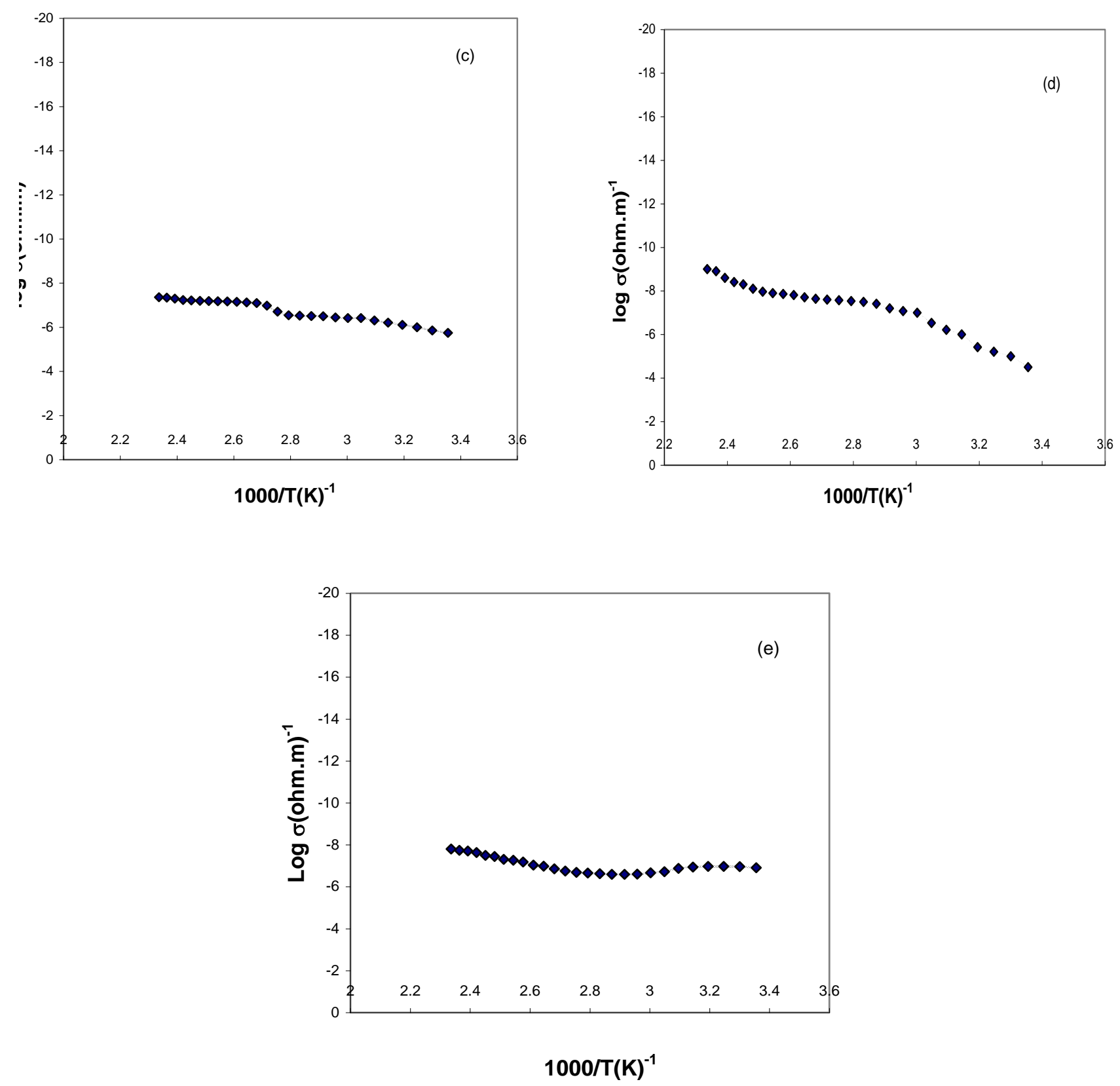

Figure 4:Variation of D.C electrical conductivity with temperature for (a) PVA/ PPY uncapped (b) PVA/ PPY composite with $\mathrm{FeCl}_{3}$ (c) PVA/ PPY composite with $\mathrm{NiCl}_{2}$ (d) PVA/ PPY composite with $\mathrm{CuCl}_{2}(\mathrm{e})$ PVA/ PPY composite with $\mathrm{ZnCl}_{2}$. 
Figure $(4: a, b, c, d, e)$ shows the variation of the conductivity with inverted temperature for PVA/ PPY uncapped and PVA/PPY with metal chlorides $\left(\mathrm{FeCl}_{3}, \mathrm{NiCl}_{2}, \mathrm{CuCl}_{2}\right.$ and $\left.\mathrm{ZnCl}_{2}\right)$ nanocomposite respectively. The electrical conductivity increases with increasing temperature indicating that, the increase can be assigned to two factors: the increase in the mobility of charge carriers and the increase in the rate of charge carrier's generation, $R$ Sing et al [14]. Also from the figures it can be noticed that, the electrical conductivity of PVA/PPY is less than PVA/PPY with metal chlorides nanocomposite. The increase in electrical conductivity $\sigma$ with added metals can be attributed to the creation of induced charge carriers in PVA/PPY matrix. This is because of the metal ions coordinated through ionic bonds with hydroxyl group belonging to the different chains in PVA and with the hydrogen in the $\mathrm{N}-\mathrm{H}$ group of the pyrrole ring, Anurag Krishna et al [15].The addition of metal increase the volume required for ionic carriers to drift in the polymer matrix. This enhances the ionic mobility and hence increases the conductivity. The dependence of electrical conductivity $\sigma$ of PVA/PPY and PVA/PPY metal nanocomposite at (393K) is shows in table (1)

Table (1): Electrical conductivity of PVA/PPy and PVA/PPy metal nanocomposite at (393K)

\begin{tabular}{|c|c|}
\hline Samples & $\sigma\left(\Omega . \mathrm{cm}^{-1}\right.$ \\
\hline PVA/PPY & $3.86 \times 10^{-9}$ \\
$\mathrm{FeCl}_{3}$ & $1.89 \times 10^{-8}$ \\
$\mathrm{NiCl}_{2}$ & $6.77 \times 10^{-8}$ \\
$\mathrm{CuCl}_{2}$ & $8.25 \times 10^{-8}$ \\
$\mathrm{ZnCl}_{2}$ & $5.39 \times 10^{-8}$ \\
& \\
\hline
\end{tabular}

The table given that the PVA/PPY with metal nanocomposite is higher conductivity than PVA/PPY. Also it can be observed the high conductivity for $\mathrm{Cucl}_{2}$ doped with PVA/PPY, this could be attributed to small polaron hopping. So the prepared PVA/PPY/ $\mathrm{CuCl}_{2}$ nano composites in the present study are expected to be more useful in photonic and electronic device applications, Alabur Manjunath et al.[16] .The results obtained in the present work is of the same order as it is reported in the literature, Ahmed hasim et al [17].

\section{Conclusion}

PVA/PPY nanocomposite with metal chlorides $\left(\mathrm{FeCl}_{3}, \mathrm{NiCl}_{2}, \mathrm{CuCl}_{2}\right.$ and $\mathrm{ZnCl}_{2}$ ) have been synthesized by chemical oxidative polymerization method. FTIR analysis indicated strong interactions between metals and PVA/PPY matrix, leading to shifting of bands to higher wavenumbers. X-ray diffraction pattern of nanocomposites show the intensity of diffraction peaks was decreased and broad. This attributed to the capped between PVA/PPY nano composites with metal decreased the crystallinity of metal chlorides. TEM images showed that the particle size of nanocomposite becomes smaller, this attributed to the metal nanoparticles are entrapped in the PVA/PPY matrix. The DC electrical conductivity of PVA/PPY/metal chlorides gradually increased with added metals, this is attributed to the creation of induced charge carriers in PVA/PPY matrix. The high conductivity for $\mathrm{CuCl}_{2}$ doped with PVA/PPY, could be attributed to small polaron hopping. So the prepared PVA/PPY/ $\mathrm{CuCl}_{2}$ nano composites in the present study are expected to be more useful in photonic and electronic device applications 


\section{Acknowledgement:}

The author would like to thank Faculty of Women for Arts, Science and Education (Ein Shams University) and Petroleum Research Institute for facilitating the measurements.

\section{Reference:}

[1]L Pan , KY Zhang, YG Li, SQ Bo, and YS Li,J.Appl.polym.sci.,104,4188,(2007).

[2] H Byun and et al,Macromol.Res,16,189, (2008).

[3]H N M Ekramul Mahmud, and Auuar Kassim, IJFPS, Vol. 1(1),28, (2011).

[4]D M Nerkar, MR Rajwade, SE Jaware and G G Padhye, Archives of Applied Science Reserch, 7(10):17,(2015).

[5]K Lee, S Cho, S h Park, A J Heeger, and C w Lee, Metallic transport in polyaniline Nature, 441 : 65, (2006).

[6]Q Adeloju, and S B, Sensor S. \& Actuators B: Chemical 106 ,541, (2005).

[7]DB Dupare, MD Shirsatand,and S Aswar, "Indian Journal of chemical Technology 18(6),446,(2011).

[8]H Bai, and GQ Shi, , Rev sensors. 7(3), 267,(2007).

[9]MF Mabrook, C Pearson, and MC petty, Sensor and Actuators B-chemical. 115, 547,(2006).

[10]DJ Shirale and et al Mater Lett. 60, 1407, (2006).

[11]T Trindade, PO Brien and NL Pickett, Chem.Mater.13,3843, (2001).

[12]Kyung Jin Lee, Jihye Lee, Jin-Yong Hong, and Jyongsik Jang, Macromolecular Research,17,476, (2009).

[13]Chivukula Srikanth, B Chakradhar Sridhar, BM Nagabhushana, and RD Mathad, Journal of Engineering Research and Applications,4,38,(2014).

[14]R Singh.,J Kumar.,R K Singh.,A Kaur and R D P Sinha.,N P Gupta,Polymer,47,5919-5928,(2006).

[15]Anurag Krishna, Amit Kumar, and Rakiv Kumar singh, ISRN Nanomaterials, vol.6, (2012).

[16]Alabur Manjunath,Mohammed Irfan,Kabbinadavamsha Prasannakumar Anushree,Kuruba Mahesha Vinutha, Narasimhappa Yamunarani, Scientific Research, vol(6)10,(2016).

[17] Ahmed hasim, H Bahaa, Majeed Ali, Nahda Abd-alkadhim, and Athraa Saad, American journal of scientific research,74,5,(2012). 